\title{
Dawn mission and operations
}

\author{
C.T. Russel ${ }^{1}$, C.A. Raymond ${ }^{2}$, T.C. Fraschetti ${ }^{2}$, M.D. Rayman ${ }^{2}$, \\ C.A. Polanskey ${ }^{2}$, K.A. Schimmels ${ }^{2}$ and S.P. Joy ${ }^{1}$ \\ ${ }^{1}$ Institute of Geophysics and Planetary Physics, University of California, Los Angeles, \\ CA 90095-1567 \\ email: ctrussel@igpp.ucla.edu \\ ${ }^{2}$ Jet Propulsion Laboratory, Pasadena, CA 91109
}

\begin{abstract}
Dawn is the first mission to attempt to orbit two distant planetary bodies. The objects chosen, 4 Vesta followed by 1 Ceres, are the two most massive members of the asteroid belt that appear to have been formed on either side of the dew line in the early solar nebula. This paper describes the present status of the mission development and the plans for operation at Vesta and Ceres.
\end{abstract}

Keywords. 1 Ceres, 4 Vesta, Dawn Discovery Mission

\section{Introduction}

The solar system is the epitome of diversity. Mercury, formed close to the Sun, is relatively small and rather dense, indicative of a large iron core covered with a thin silicate crust. Venus, a much larger planet, has a thick atmosphere, is possibly still volcanically active, and is dominated by sulfur chemistry. It too has an iron core and silicate mantle. The Earth provides a much more benign habitat for life, with water on the surface. While the interior may be similar to that of Venus, plate tectonics acts to move the crust and to enhance heat flow from the interior. Mars size lies between Mercury and the Venus-Earth twins. While its weak atmosphere, low temperature and low gravity support neither the sustained presence of surficial liquid water, nor much water vapor in the atmosphere, Mars apparently possesses much subsurface water, at least initially resulting in episodic, wet epochs and significant erosion of the surface. The gas giants, Jupiter and Saturn, present an entirely different style of planet, with relatively small rocky cores, rings and many moons (mostly icy). Uranus and Neptune are significantly smaller than Jupiter and Saturn and are believed to consist of a rocky core surrounded by a water mantle and a hydrogen and helium envelope. They have weak ring systems and multiple moons, again icy. The edge of the solar system brings us Pluto, the Kuiper belt and the Oort cloud, frozen remnants from the earliest days of the solar system.

The planets retain an unsatisfactory record of the early solar system. Their compositions represent an average over a significant radial range in the solar nebula and they have undergone much thermal evolution, continuing to the present time. The comets and Kuiper belt objects also provide an unsatisfactory record of the solar nebula because their present orbits have been greatly altered by the gas giants. Thus it is difficult to relate the measurements of today to the events of yesterday.

The asteroid belt forms a transition from the rocky terrestrial planets to the wet and icy outer solar system. It has been affected by the gravitational stirring of Jupiter that stopped the growth of the planetary embryos in the belt, and that spawned a destructive collisional environment. The early cessation of growth enables us to explore backward 


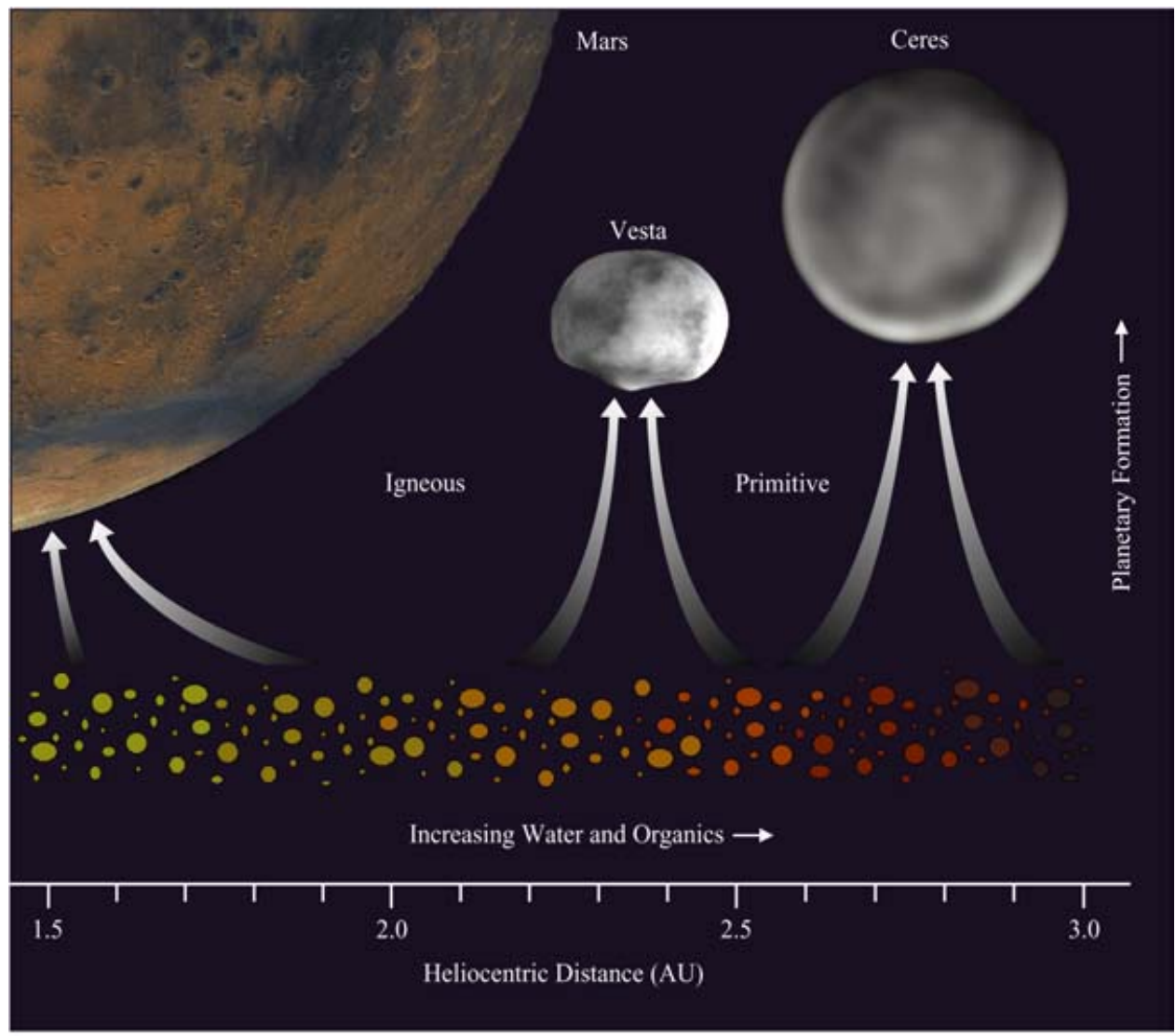

Figure 1. Compositional gradient of solar nebula leads to variation in properties of accreted bodies as a function of heliocentric distance.

in time. Smaller objects evolve less than the larger planets and preserve information on much earlier epochs. The growth of the minor planets stopped early, probably well before the formation of the Earth. The collisions produced by disruptive stirring in the belt are a potential impediment to exploration backward in time. They may have destroyed many of the smaller embryos or scattered them far out of their original orbits. Thus the best candidates for study are the largest asteroids. These objects survived the heavy bombardment and they are the most likely to be near their place of origin. If we wish to determine the original heliocentric gradient, then we should visit the largest asteroids. This strategy underlies the Dawn mission.

The two most massive minor planets are 1 Ceres and 4 Vesta. Figure 1 illustrates their size relative to that of Mars. They clearly merit being labeled planets as they resemble the other planets in many ways. Further Vesta and Ceres differ from each other in a manner not simply explainable by their relative size. They arose at different distances from the Sun at which the composition of the dusty solar nebula was quite different. The differences in the spectrum of reflected light from the surfaces of Vesta and Ceres indicates a significant gradient in the properties of the early solar nebula when the asteroids formed. If we wish to constrain the gradient in the early solar nebula, where should we explore? Figure 2 shows a sketch of the relative locations and relative sizes of the larger minor planets. The distance from the ecliptic plane on this plot is a qualitative indication of both the eccentricity and inclination of their orbits. Five minor planets are most worthy 


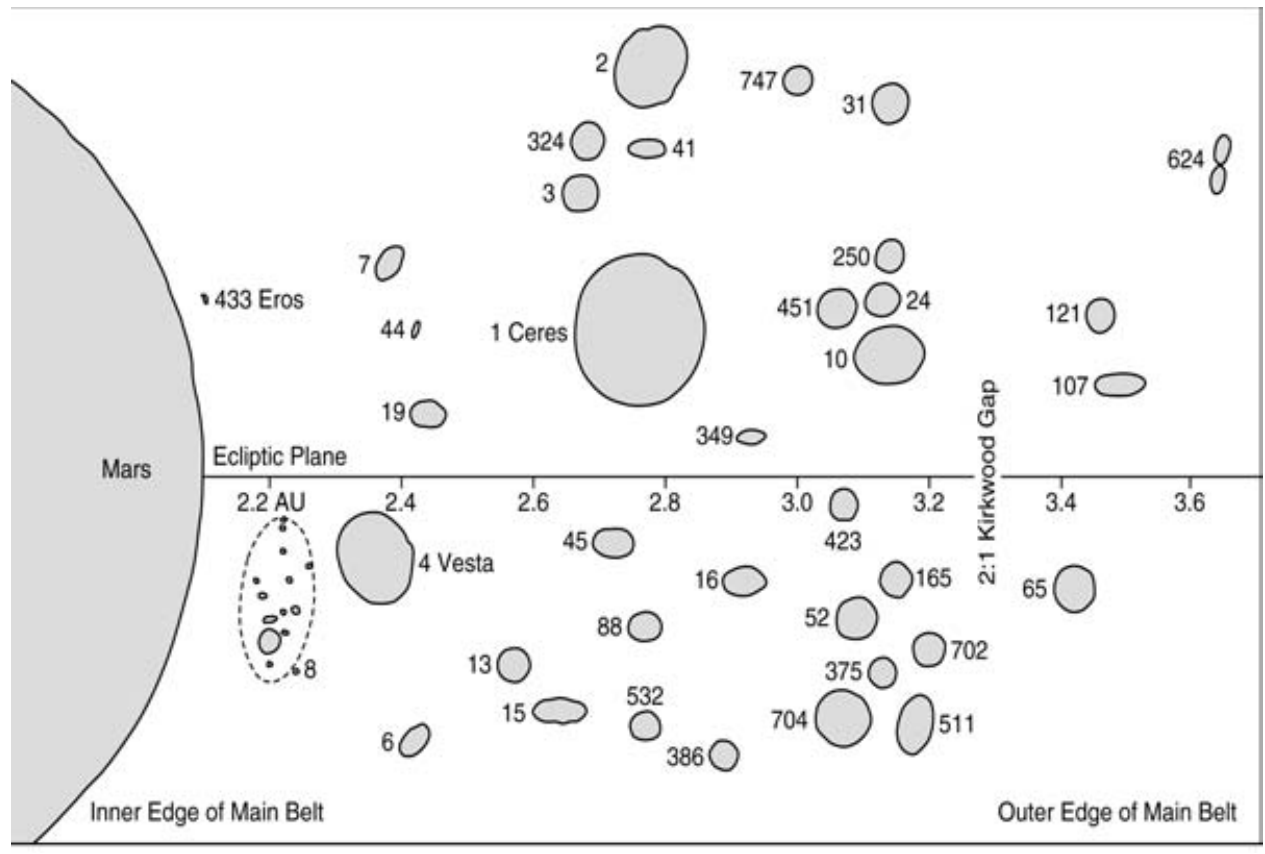

Figure 2. Qualitative sketch of the location and relative sizes of the larger minor planets. The distance from the ecliptic plane is greater for bodies with larger inclination or eccentricity and qualitatively reflects the difficulty in reaching these bodies as compared with others at the same semimajor axis.

of our attention: 1 Ceres, 4 Vesta, 3 Pallas, 10 Hygiea and 16 Psyche. Table 1 lists their semi-major axes, inclinations and masses. These five objects give us a wide range of asteroid types. Ceres is a rocky planet with probably a thick icy crust (McCord \& Sotin 2005). Vesta is a rocky body, similar to the Moon, with an iron core and a silicate mantle. Pallas may be very similar to Ceres, but is significantly smaller. Little is known about Hygiea, but, being much further from the Sun than Ceres (and Pallas), it is expected to differ markedly from Ceres. Interest is added by its dynamical family of quite diverse membership, including $\mathrm{B}, \mathrm{C}, \mathrm{D}$ and $\mathrm{S}$ type asteroids that might be accessible during a Hygiea mission. Finally, Psyche has all the indications of being an iron core of an originally much larger parent body. It reflects visible light and radar waves like iron does and it has a bulk density close to that of iron (Kuzmanoski \& Kovacevic 2002).

It is certainly possible to visit such asteroids with chemical rockets but affordable missions would entail flybys. To make the needed physical, geochemical, geophysical, and geological measurements we need to orbit these bodies. We do not have to visit all the asteroids to determine the nebular gradient, but we do need to visit at least a handful. The problem is to do this affordably.

\section{The Mission}

\subsection{Ion Propulsion: The Enabling Technology}

An affordable solution to exploring the minor planets is provided by ion propulsion. Since planetary missions are governed by Keplers laws of planetary motion regardless of how they are propelled, and since ion thrusters do not accelerate spacecraft much above the Keplerian velocities, there is a long time over which the weak thrust of ion propulsion can 


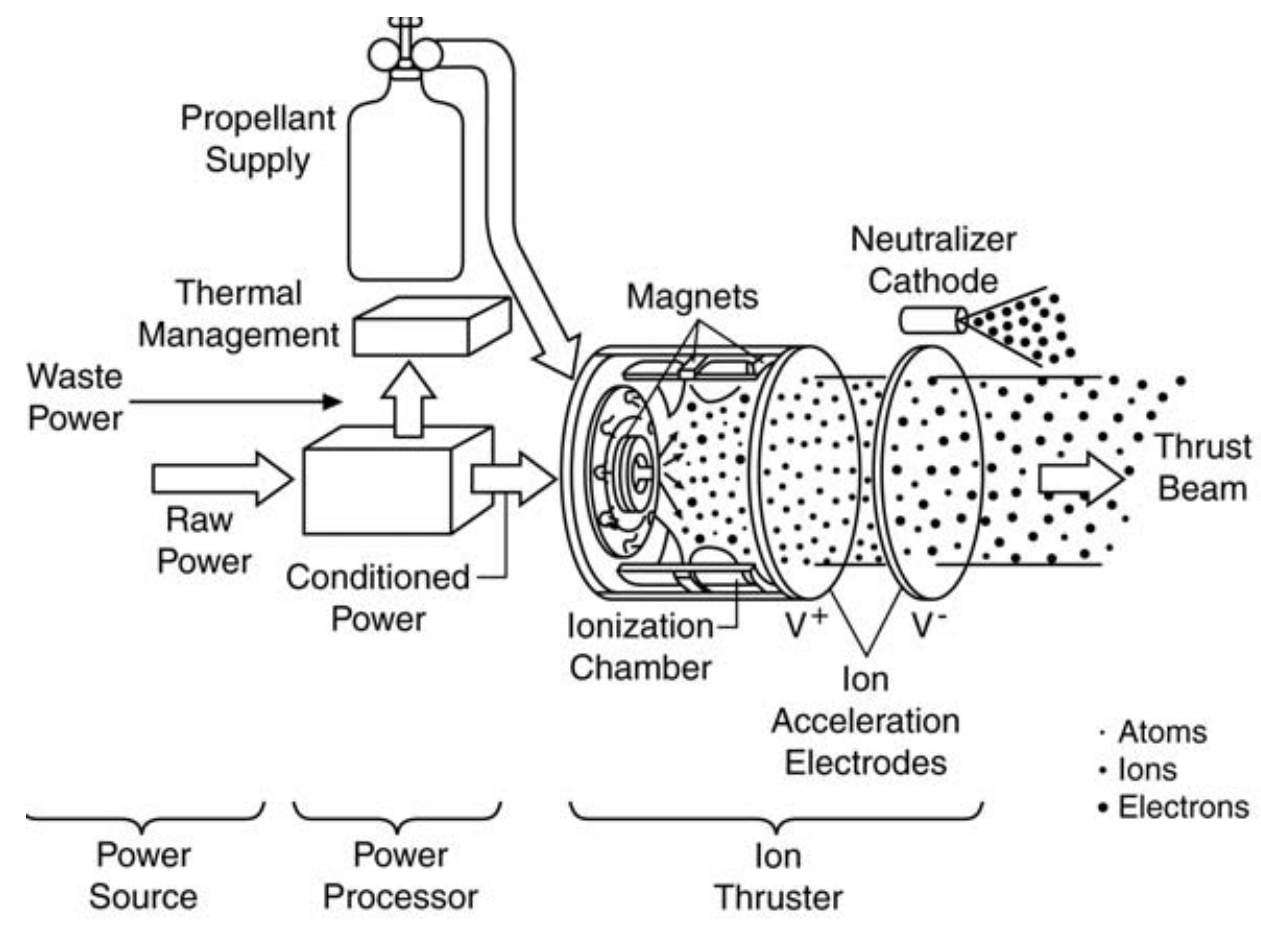

Figure 3. Operating principle of an ion engine.

act. The thrusters accelerate the ions to a very large velocity, currently about $35 \mathrm{kms}^{-1}$, an order of magnitude greater than the velocity of the fuel expelled by a chemical engine. Thus the fuel carried on board is used with great efficiency. Once in deep space the ion engine (whose thrust is far too weak to lift the spacecraft off the planet) can change the velocity of the spacecraft (after years of thrust) by an amount equal to that provided by the launch vehicle that lifted the spacecraft off the surface of the Earth $\left(11 \mathrm{kms}^{-1}\right)$.

The general principles of an ion engine are shown in Figure 3. An ion engine requires much electric power, provided at present by solar arrays. A typical solar-power-driven ion engine requires arrays providing about $10 \mathrm{~kW}$ at $1 \mathrm{AU}$ for exploring the asteroid belt. This power is used to ionize the xenon fuel and to accelerate the ions to $35 \mathrm{kms}^{-1}$, resulting in a thrust of about $90 \mathrm{mN}$. In order that the ions can escape from the spacecraft, it must not accumulate charge. To maintain charge neutrality electrons are injected into the ion beam. Xenon is used as fuel because it is environmentally benign, can be stored in space as a gas, and is readily ionized. Design of the grids is a special concern as the accelerated ions ablate the grid material limiting the lifetime or throughput of the engine. A single, present-day thruster is rated to process about $150 \mathrm{~kg}$ of xenon. Thus if a mission requires $300 \mathrm{~kg}$ of xenon it might carry two thrusters. If redundancy were required a third thruster might be included. Other considerations include providing gimbals to adjust the direction of thrust to keep the thrust vector directed through the center of mass of the spacecraft to minimize torques, and the design of the power processing units and power distribution units needed to handle the significant power that such spacecraft require. 
Table 1. Linear correlation coefficients for the data sets plotted in figure 1

\begin{tabular}{llccc}
\hline Name & Mass [kg] & Distance [AU] & Diameter $[\mathrm{km}]$ & Comments \\
\hline 1 Ceres & $9.4 \times 10^{20}$ & 2.77 & $974 \times 909$ & $\sim 100 \mathrm{~km}$ water-ice mantle \\
4 Vesta & $2.7 \times 10^{20}$ & 2.34 & $570 \times 458$ & $\sim$ up to 250 km diameter iron core \\
2 Pallas & $2.4 \times 10^{20}$ & 2.77 & 523 & A smaller Ceres? \\
10 Hygiea & $1.0 \times 10^{20}$ & 3.14 & 407 & Dynamical family includes D,B,C,S types \\
16 Psyche & $0.7 \times 10^{20}$ & 2.90 & 264 & $\begin{array}{c}\text { Density and reflectivity of iron; could be } \\
\text { core of larger parent body }\end{array}$ \\
\hline
\end{tabular}

\subsection{Choosing a Target}

Table 1 lists five possible choices: a rocky asteroid about which we know much from the Howardite, Eucrite and Diogenite meteorites (HEDs); the most massive minor planet that appears to be very wet, and remains very much an enigma; a high inclination body that may also be wet and is even more of an enigma; a distant asteroid with an interesting dynamical family (some of which may be adopted); and an asteroid that very much resembles the core of a larger body, stripped of its rocky mantle. Four of these bodies we could probably visit with a present-day, ion-propelled missions, affordable under NASAs Discovery program guidelines. However, it is very difficult to reach the high inclination orbit of Pallas, so it appears to be presently out of contention as a Discovery candidate. It is also not possible to visit all four asteroids with a single spacecraft. The impossibility results from in part the long durations of such a multiple asteroid mission. It is simply too costly to operate for such a long period. This exceeds Discovery cost guidelines, and the spacecraft would require very high reliability to last that long, another cost driver. The spacecraft would have to be quite large with multiple thrusters (six?) and a large xenon load (750 kg?). A relatively inexpensive rocket, like the Delta II, would be unable to launch such a mission to escape velocity. Thus, we must lower our sights.

The idea of multiple rendezvous with two or more asteroids has long intrigued the Dawn team (Russell et al. 2004). By the year 2000, the capability of the ion thrusters and the celestial position of Vesta and Ceres had evolved sufficiently to allow a single spacecraft powered by three ion engines, used one at a time, to rendezvous with both Vesta and Ceres. This mission was proposed to NASA and selected for flight in 2001 (Step 1 selection in January and Step 2 in December). The tortuous path to confirmation is described by Russell et al. (2005). Figure 4 shows a schematic diagram of the mission trajectory. The spacecraft is launched in summer of 2006; it flies by Mars in 2009; and reaches Vesta in 2011, and Ceres in 2015, with slightly under a year in orbit at each body.

\subsubsection{Vesta}

The fourth minor planet, 4 Vesta, was discovered on March 29, 1807 by H. Olbers. Much, much later it was realized that both the reflectance spectrum of Vesta resembled that of a basaltic surface, and that a class of meteorites had that same reflectance spectrum (McCord et al., 1970). Pieces of Vesta (perhaps with the intermediary of Vestoids, collisional fragments from Vesta) had been falling on the Earth. In fact one in twenty falls are of this class, the Howardite-Eucrite-Diogenite (HED) meteorites. Since this discovery many observations have supported this interpretation. Our best estimate of the shape of Vesta is a triaxial ellipsoid with radii 289,280 and $229 \pm 5 \mathrm{~km}$. Its mass is $2.71 \pm 0.10 \times 10^{20} \mathrm{~kg}$ corresponding to a density of $3750 \pm 400 \mathrm{~kg} / \mathrm{m}^{3}$. It has a large southern crater as shown in Figure 5. Evidence for a significant iron core has been obtained from the HED meteorites. HST and adaptive optics observations reveal a non-uniform surface with significant variations in composition (Binzel et al. 1997; Drummond et al. 


\section{Mission Itinerary}

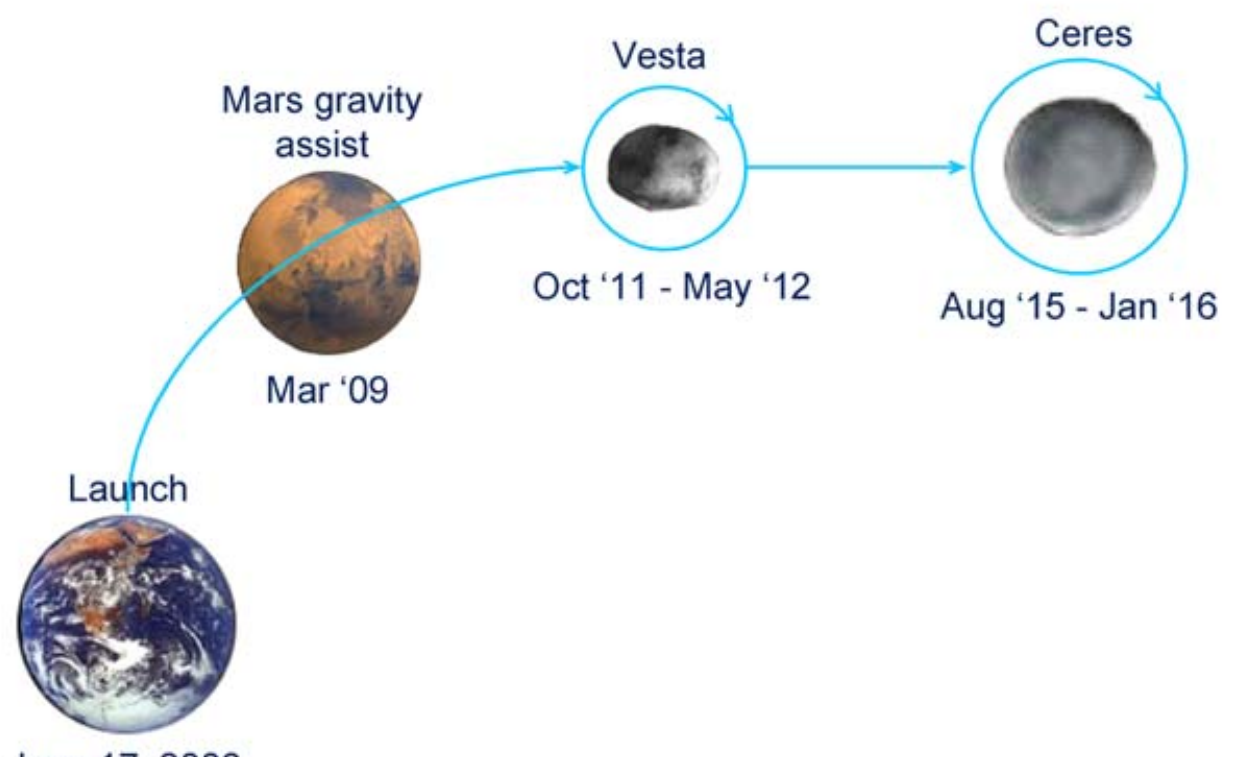

June 17, 2006

Figure 4. Schematic diagram of mission trajectory.

1998). We presume that these variations are caused by both cratering exposing different materials at depth and lava flows that were extruded at different times and from different reservoirs during Vestas thermal evolution. Since Vesta was formed in the solar nebula near the dew or frost point, the presence of water in or on Vesta remains an interesting question. It is clear from the HED meteorites that Vesta is very dry and that water did not much influence its evolution. However, the discovery of a quartz veinlet in a HED meteorite (Treiman et al. 2004) makes a strong case for liquid water at least transiently on Vestas surface. A more thorough review of our knowledge of Vesta has been given by Russell et al. (2005).

\subsubsection{Ceres}

1 Ceres was discovered on January 1, 1801 by C. Piazzi. In great contrast to Vesta there are no known Ceres-associated meteorites and there are no known Ceroids, collisional fragments of Ceres. This immediately raises the question as to whether the crust of Ceres is rock or some material such as water-ice that would not be stable if removed from Ceres gravitational potential well and the expected dust covering the surface. In fact McCord \& Sotin (2005) have constructed a thermal evolution model of Ceres that results in a rocky core covered with an approximately $100 \mathrm{~km}$ thick water and ice mantle that may or may not freeze completely in 4.6 billion years.

The mass of Ceres is $9.43 \pm 0.05 \times 10^{20} \mathrm{~kg}$ with a shape of an oblate spheroid of $487 \pm 2 \mathrm{~km}$ (equatorial) and $455 \pm 2 \mathrm{~km}$ (polar) (McCord \& Sotin 2005; Thomas et al. 2005). Given its $9.075 \mathrm{hr}$ spin period this shape corresponds to a non-uniform, radial distribution of 


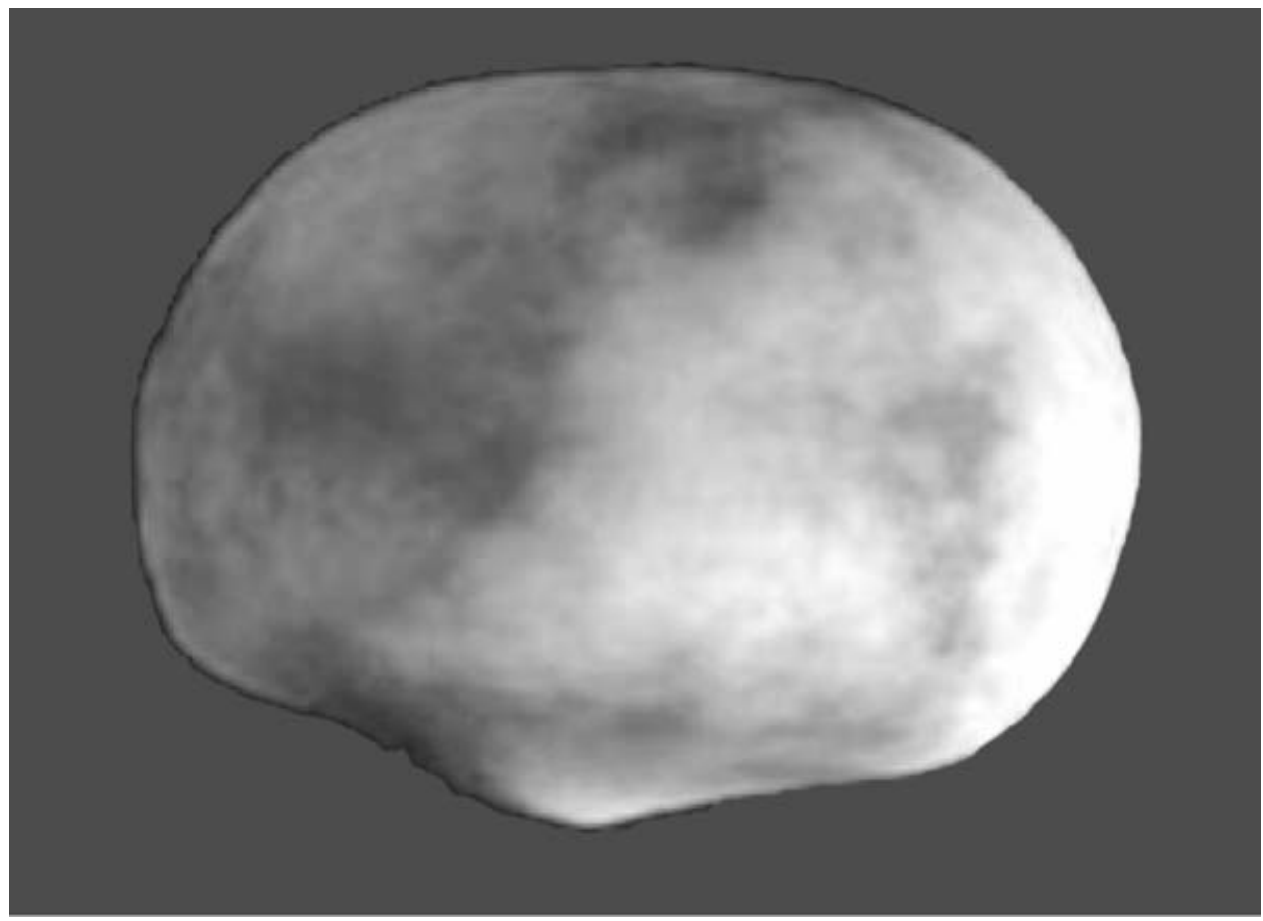

Figure 5. Image made from a computer model of Vesta.

mass, consistent with about $100 \mathrm{~km}$ of water ice on top of about $400 \mathrm{~km}$ of rock. The same HST observational campaign that produced the shape observations also produced albedo maps at $223 \mathrm{~nm}, 335 \mathrm{~nm}$ and $535 \mathrm{~nm}$ (Li et al. 2005). A preliminary color map of Ceres surface at $535 \mathrm{~nm}$ is shown in Figure 6. Ceres has well defined surface features that can be tracked, enabling the rotation rate and pole position to be determined. However, the average albedo is low and the contrast across the surface small. The surface is smooth at radar wavelength scales and as revealed in the Hapke parameters. However, it is rough at intermediate wavelengths. At planetary scales, as stated above, the body is relaxed to a shape that is in rotational equilibrium, consistent with the presence of a rocky core and a water-ice mantle.

\subsection{Science Objectives}

Dawn's main objective is to achieve an understanding of the conditions and processes acting at the solar systems earliest epoch. To do this the mission collects images of the surface of Vesta and Ceres and thereby determines their bombardment, thermal, tectonic and possible volcanic history. Dawn determines the topography and internal structure of the two bodies by measuring their mass, shape, volume and spin state with navigation data and imagery. It determines mineral and elemental composition from infrared, gamma ray, and neutron spectroscopy to constrain the thermal history and compositional evolution of Vesta and Ceres. It also provides context for the HED meteorites, samples of the surface already in hand.

These science objectives are allocated to each of the three instruments (Framing Camera, Visible and IR Mapping Spectrometer, and the Gamma Ray and Neutron Spectrometer) and to the gravity science investigation. This allocation in turn levies specific 


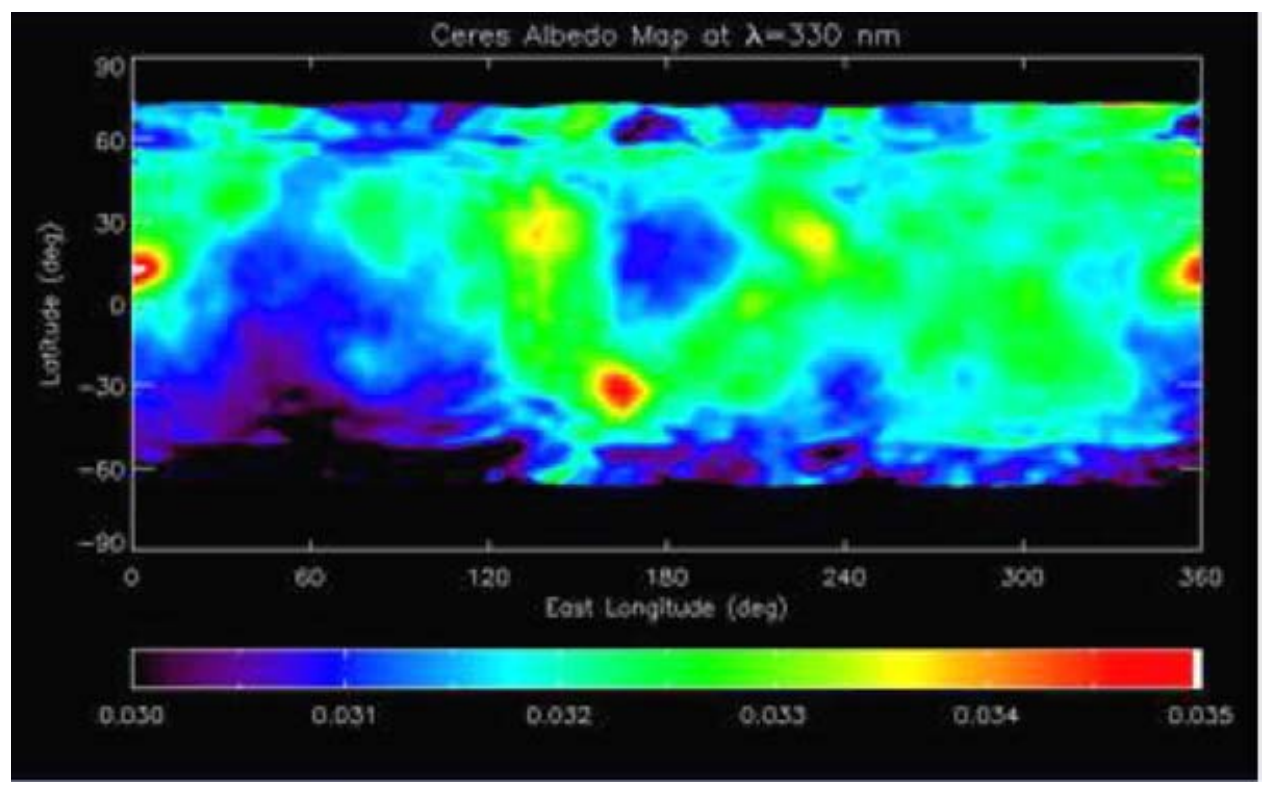

Figure 6. HST albedo map of Ceres (Courtesy of J-Y. Li, 2005).

measurement requirements. In particular the mission is attempting to measure the bulk density of Vesta and Ceres to better than 1\% their spin-axis orientations to better than $0.5^{\circ}$, and Vestas gravity field to better than $90 \mathrm{~km}$ half-wave length resolution (better than $300 \mathrm{~km}$ for Ceres). Over $80 \%$ of the surfaces of both bodies will be imaged at better than $100 \mathrm{~m}$ per pixel for Vesta and better than $200 \mathrm{~m}$ per pixel at Ceres in 3 or more color filters. A topographic map of the surfaces of both bodies will be made with a goal of $10 \mathrm{~m}$ vertical accuracy on Vesta and $20 \mathrm{~m}$ on Ceres. The mission attempts to map the abundances of the major rock forming elements to $\pm 20 \%$ precision with a spatial resolution of $\sim 1.5$ times the mapping altitude, and to map the abundances of hydrogen and the radioactive elements $\mathrm{Th}, \mathrm{U}$ and $\mathrm{K}$. The visible and infrared mapping spectrometer will map the mineral composition with spectra between 0.25 and 5 microns with a resolution of 2 to $10 \mathrm{~nm}$. Half of the frames will have a resolution of better than $200 \mathrm{~m}$ on Vesta and $400 \mathrm{~m}$ on Ceres. Dawn also uses its radiometric tracking system to measure the gravitational field.

\subsection{Scientific Payload}

\subsubsection{Framing Camera}

The two redundant framing cameras (FC) were built by MPS in Lindau, Germany, in cooperation with DLR Berlin and IDA, Braunschweig. The camera shown in Figure 7 uses a $f: 1 / 8$ radiation-hard refractive optics with a focal length of $150 \mathrm{~mm}$. The field of view of $5.5^{\circ}$ by $5.5^{\circ}$ is imaged by a frame-transfer CCD with 1024 by 1024 pixels for a spatial resolution of $9.3 \mathrm{~m} /$ pixel at $100 \mathrm{~km}$. A data processing unit controls the camera and handles the compression and buffering of the data. Compression rates vary from $2: 1$ (lossless) to $10: 1$ (lossy). A filter wheel provides 1 clear filter and 7 spectral windows. A more detailed description has been provided by Russell et al. (2005).

\subsubsection{Visible and Infrared Mapping Spectrometer}

The mapping spectrometer (VIR) has been provided by the Italian Space Agency and the National Institute for Astrophysics. It combines two data channels in one compact 


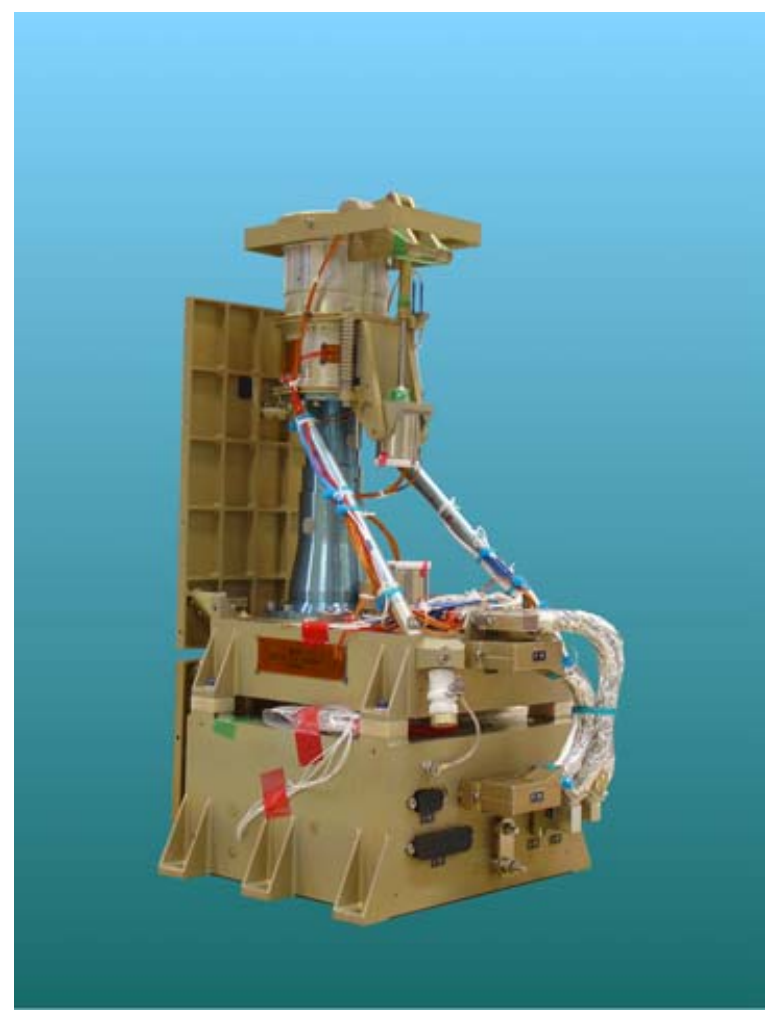

Figure 7. One of two redundant framing cameras.

instrument. The visible channel covers 0.25 to 1 micron while the infrared channel covers 1 to 5 microns. The use of a single optical chain and overlap in wavelength between two channels facilitates intercalibration. The VIR instantaneous field of view is $250 \mathrm{~m} / \mathrm{pixel}$ at $100 \mathrm{~km}$ while the full field of view is $64 \mathrm{mrad}$. Figure 8 shows the VIR instrument. The infrared focal place array is cooled to an operating temperature of $70 \mathrm{~K}$ by a Stirling cooler. Rice lossless data compression is used to return data with a compression factor of between 2 and 5 . Further details on the VIR instrument have been provided by Russell et al. (2005).

\subsubsection{Gamma Ray and Neutron Spectrometer}

The Los Alamos National Laboratory provided the gamma ray and neutron spectrometer (GRaND). The gamma-ray sensor is segmented into two parts and the neutron sensor into four parts. On board classification of the multiple signals from each event that can discriminate radiation from the asteroid and from the spacecraft. The gamma-ray sensor is bismuth germanate. Cadmium Zinc Telluride sensors provide a new redundant technology for sensing the gamma rays. Upward (asteroid) and downward (spacecraft) facing boron-loaded plastic segments are optically coupled to a $6 \mathrm{Li}$-loaded glass scintillator to detect neutrons. Figure 9 a shows a picture of the instrument. Further details may be found in Russell et al. (2005).

\subsection{Spacecraft}

The flight system is built by Orbital Sciences Corporation with oversight and direction by the Jet Propulsion Laboratory. Figure $9 \mathrm{~b}$ shows the spacecraft. It is large, $19.7 \mathrm{~m}$ from tip to tip, three axis stabilized, keeping its solar panel axis perpendicular to the 


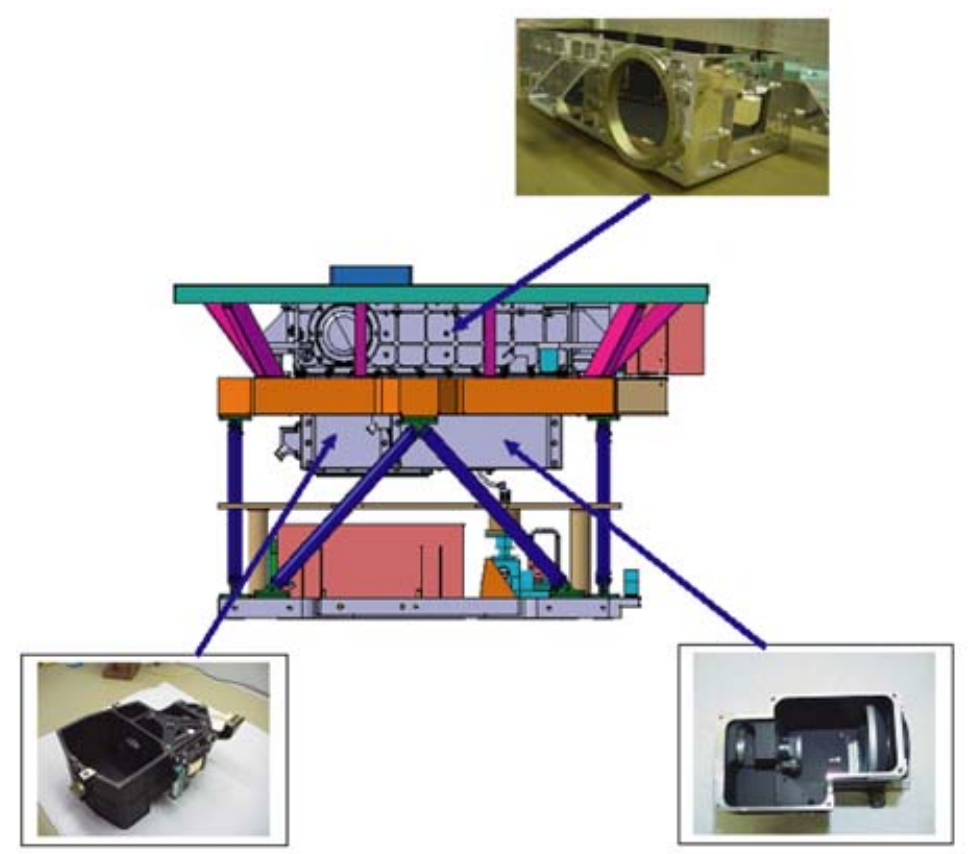

Figure 8. Mapping spectrometer, showing specific subsystems and their location in the instrument.

solar direction and the arrays normal to the star direction. The instruments look in the nadir direction (top of the spacecraft here) that, while in orbit, is kept facing the planet. The main structure of the spacecraft is approximately $1.4 \mathrm{~m}$ on a side. The solar panels generate about $10 \mathrm{~kW}$ of power at $1 \mathrm{AU}$. The spacecraft carries $425 \mathrm{~kg}$ of xenon. The high gain antenna is mounted on the body of the spacecraft and the spacecraft rotates so that the antenna points at Earth during telemetry sessions.

\section{Operations}

\subsection{Science Observation Strategies}

Dawn achieves its science objectives by orbiting the main belt asteroids (protoplanets) 1 Ceres and 4 Vesta. Dawn will orbit Vesta for a period of not less than seven months and Ceres for not less than five months, of which at least 1.5 months at Vesta will be below a mean altitude of $200 \mathrm{~km}$, and one month at Ceres will be below $700 \mathrm{~km}$ mean altitude.

The use of ion propulsion results in a highly flexible mission plan, with a long launch period. Dawns launch period opens in the summer of 2006 and is determined by project readiness. The launch period lasts three weeks, which is defined by cost constraints and specification of accurate targeting of the vehicle; however, the acceptable launch period lasts well into the fall of 2006. Dawn will arrive at Vesta in late 2011, leave Vesta mid2012, and arrive at Ceres in the latter half of 2015, where it will remain. The spacecraft flies by Mars in 2009 enroute to Vesta. A high-level mission timeline is shown in Figure 10. The instruments on the spacecraft are body mounted so the spacecraft rotates to point the instruments at the targets. All data are stored on board for playback to Earth, and in general, only gravity science (Doppler and range measurements) will be collected during tracking sessions. All data are transmitted to Earth within a few days of acquisition. 


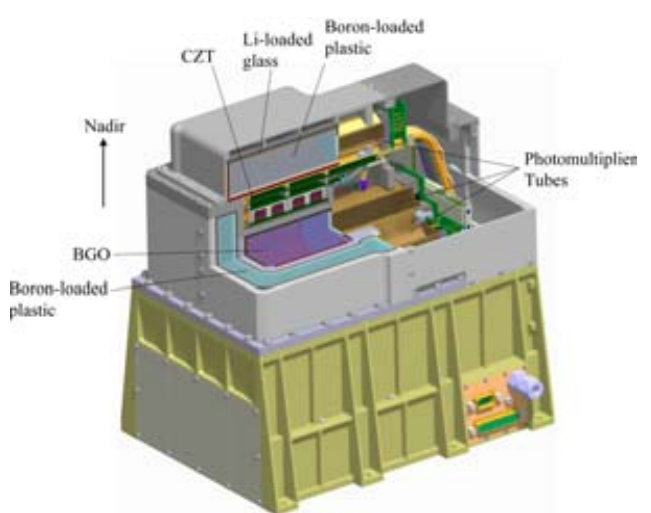

(a)

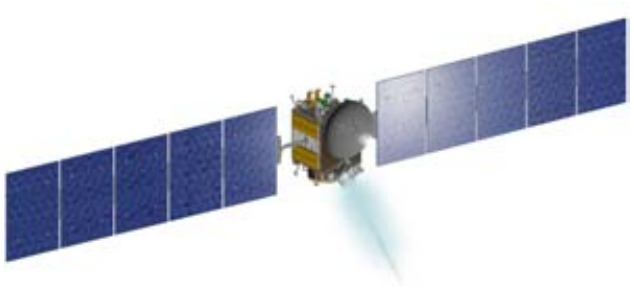

(b)

Figure 9. (a): Gamma ray and neutron spectrometer. (b): Dawn spacecraft.

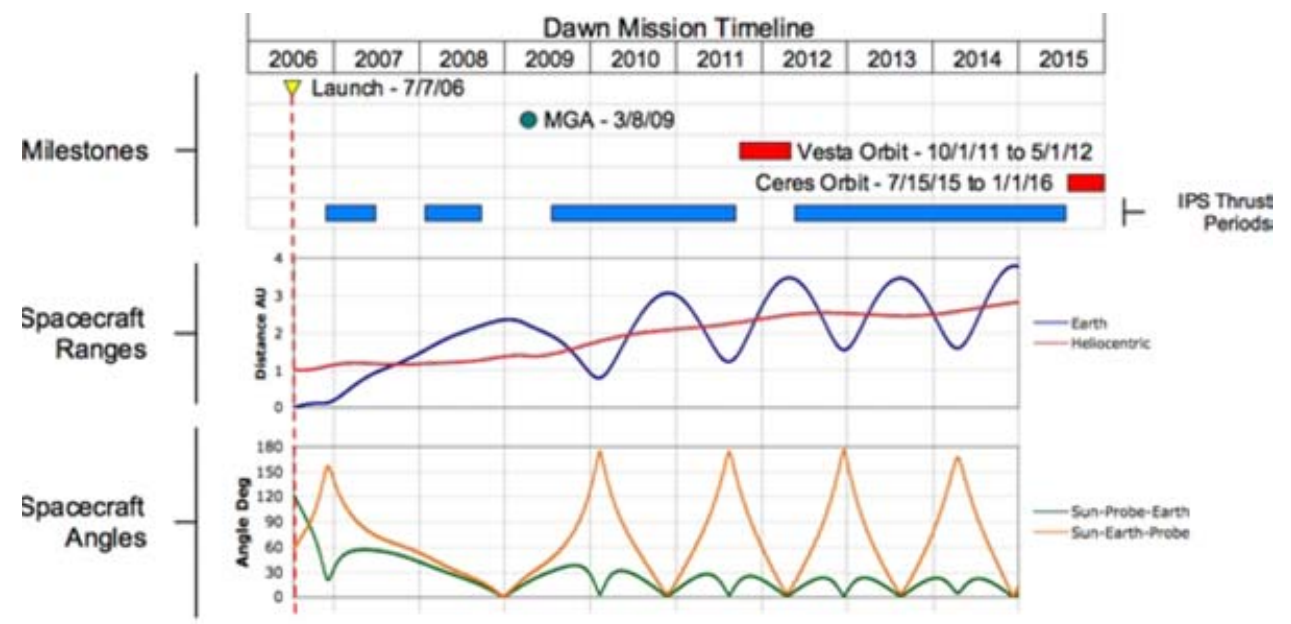

Figure 10. Dawn Mission Timeline.

\subsubsection{Mission Phases}

The mission is divided into phases, including Initial Checkout (Launch +1 to $\mathrm{L}+60$ days), Interplanetary Cruise Phases, and Mars Gravity Assist (closest approach 100 days to $\mathrm{CA}+30$ days), as well as the asteroid encounters (Science Orbits). Each Science Orbit phase begins with an Approach sub-phase starting 85 days before capture. The Science Orbit sub-phases will be: Survey Orbit, High Altitude Mapping Orbit (HAMO), and Low Altitude Mapping Orbit (LAMO). Table 2 describes the orbit sub-phases at Vesta in terms of their durations, beta angles (angle between Sun-body vector and the orbit plane), radii and periods. The minimum mission (performance floor) for the Dawn mission reduces the time spent at Vesta and Ceres from 7 months and 5 months, respectively, to 4 months at each. At Vesta, the lowest altitude orbit is eliminated, and the higher-altitude mapping orbit is lengthened to at least 30 days. At Ceres, the higher-altitude mapping orbit is lowered to $1380 \mathrm{~km}$ and lengthened to at least 30 days. 
Table 2. Vesta Orbit Sub-phases

\begin{tabular}{|c|c|c|c|c|}
\hline Sub-phase & $\begin{array}{c}\text { Duration } \\
\text { (days) }\end{array}$ & $\begin{array}{c}\text { Beta } \\
\text { Angle } \\
\text { (deg) }\end{array}$ & $\begin{array}{c}\text { Orbit } \\
\text { Raidus } \\
(\mathrm{km})\end{array}$ & $\begin{array}{c}\text { Orbit } \\
\text { Period } \\
\text { (hrs) }\end{array}$ \\
\hline Transfer from Vesta Arrival to Survey Orbit & 15 & & & \\
\hline Vesta Survey Orbit & $\begin{array}{c}7 \\
(3 \text { orbits })\end{array}$ & 30 & 2700 & 58.0 \\
\hline Transfer from Survey Orbit to HAMO & 21 & & & \\
\hline Vesta HAMO & $\begin{array}{c}26 \\
\text { (52 orbits) }\end{array}$ & 40 & 950 & 12.1 \\
\hline Transfer from HAMO to LAMO & 30 & & & \\
\hline Vesta LAMO (includes planned schedule margin) & 80 & 65 & $\sim 450$ & 4.1 \\
\hline Vesta Departure (including HAMO-2) & 68 & & & \\
\hline TOTAL & 247 & & & \\
\hline
\end{tabular}

The current plan allows an eight-month stay at Vesta, exceeding the baseline mission, that is enabled by harvesting of conservative technical margins (mass, power) as the flight system becomes well-defined. This trajectory is called the MGS_Enhanced mission. Flexible mission design enabled by the use of ion propulsion allows continual evolution of the trajectory to reflect actual performance.

\subsubsection{Activities by Mission Phase}

The Initial Checkout (ICO) mission phase is used to turn on and perform initial checkout of the instruments using ground-in-the-loop commanding. Instrument checkout during the 60-day period following launch will not be exhaustive; only a minimal set of checkout activities will be performed during the ICO to minimize interference with critical spacecraft checkouts. Two days have been set aside for Earth-Moon imaging if the contamination environment is deemed sufficiently benign to allow it. Initial instrument calibration tests are done during the early Cruise phase, following ICO.

Seven days of coast (non-thrusting periods) per year have been designated for science calibration activities. These periods will be used to perform functional, performance and calibration tests of the instruments using stellar and planetary targets. The seven days will be split into two 3.5-day periods at six-month intervals. The Framing Camera will perform a functional test each time and an extended calibration test once a year. VIR will do calibrations at each opportunity using stars, star clusters, planets, and planetary nebulae. GRaND may take the opportunity to do adjustments and anneal the instrument.

The purpose of the MGA is to add energy to the spacecraft trajectory to ensure adequate mass and power margins for the designed trajectory. As the flight system matures, and as knowledge of the actual spacecraft mass improves, margin requirements decrease. The MGA may no longer be needed and a direct trajectory to Vesta may be adopted, if this approach is consistent with the required margins.

Science data will be collected during the Approach sub-phase, and in the Science Orbit sub-phases of each encounter, to satisfy the measurement requirements specified above. The Survey Orbit sub-phase will provide an overview of the asteroid and provide 


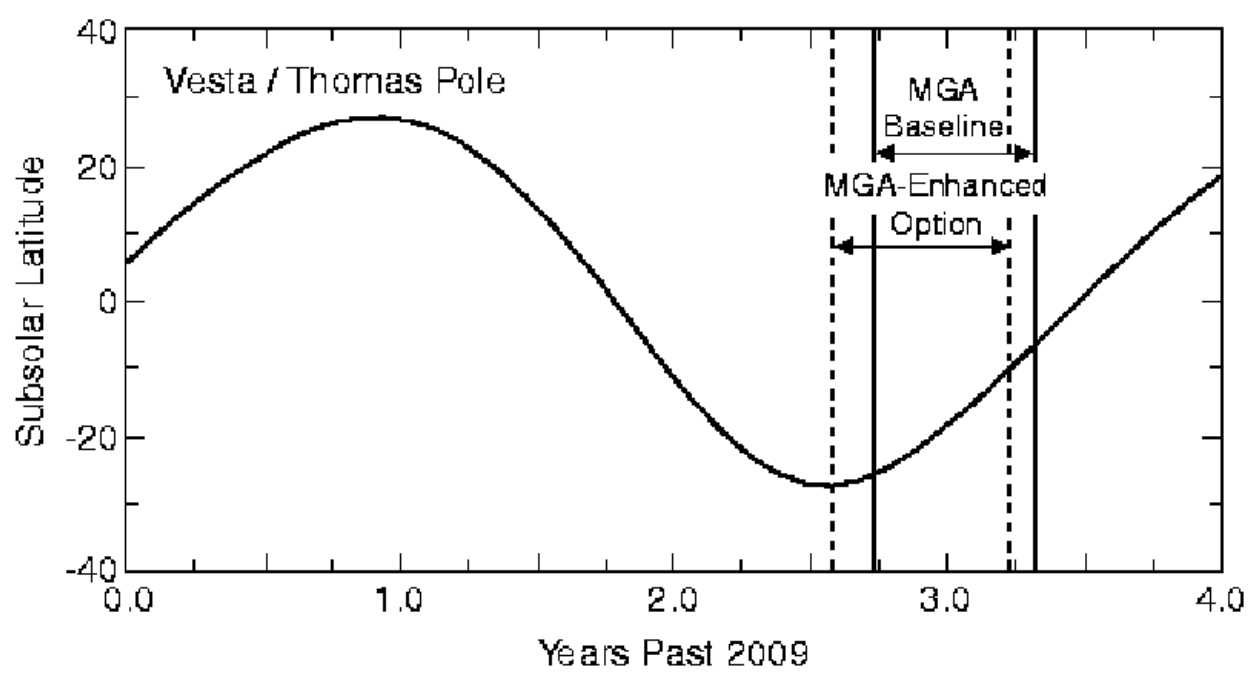

Figure 11. Vesta lighting conditions for baseline and enhanced MGA trajectories

additional observations for the planning of subsequent sub-phases. The Level 1 science required in Survey orbit is 5000 VIR spectral frames. The 5-day Survey Orbit sub-phase begins at the end of the Vesta Approach sub-phase, when the spacecraft has established a circular polar orbit at a radius of $2700 \mathrm{~km}$.

The High Altitude Mapping Orbit (HAMO) will primarily be used for the optical mapping and reflectance spectral sampling of the surface of the asteroid. The required Level 1 science for this sub-phase will be multi-color surface maps (clear and 3 filters) of $80 \%$ of the surface, a topographical map of $80 \%$ of the surface, and 5000 VIR spectral frames. This sub-phase will last approximately 26 days and will start at the conclusion of the transfer from Survey Orbit to HAMO, when the spacecraft has achieved a circular near-polar orbit at $950 \mathrm{~km}$ radius. A second HAMO sub-phase (HAMO-2) will take place during departure, to acquire images of areas that have become illuminated since the first HAMO mapping, and to fill in gaps in coverage.

The Low Altitude Mapping Orbit (LAMO) is the final science sub-phase at Vesta and will primarily be used to collect gamma ray and neutron spectra as well as to determine the gravity field. LAMO will last 80 days at Vesta and will begin at the end of the HAMO to LAMO transfer when the spacecraft is in a $460 \mathrm{~km}$ radius circular near-polar orbit. Details of the Vesta Orbit sub-phases are given in Table 2, and the lighting conditions are shown in Figure 11.

\subsubsection{Vesta Approach}

During the Approach sub-phase the instruments will go through complete calibration, repeating some of the activities that were done during the post-launch checkout calibration period, including annealing GRaND if necessary. Following the calibration activities, the FC will collect rotation characterization ( $\mathrm{RC}$ ) maps that include nadir and off-nadir viewing, that will be obtained at increasing resolution as the spacecraft approaches Vesta. For the particular trajectory used in this exercise, the phase angle (Sun-Vesta-Probe [Dawn] angle, SVP) varies from $\sim 88^{\circ}$ for the first rotation map, $(32 \mathrm{~km} / \mathrm{px})$ to $\sim 108^{\circ}$ for the second rotation map $(6.5 \mathrm{~km} / \mathrm{px})$, and captures the minimum phase angle of $\sim 42^{\circ}$ in the final, highest resolution $(\sim 0.5 \mathrm{~km} / \mathrm{px})$ rotation map. 
To create the global maps, one frame of FC data is obtained every $10^{\circ}$ of rotation of Vesta. VIR will obtain full-disc spectra coincident with the rotation characterization maps (RCs). Additionally, VIR data are desired at nadir attitude when the polar regions are visible, and the phase angle is below $90^{\circ}$. The three $\mathrm{RC}$ maps will be acquired, respectively, when the imaging is at twice the resolution of the Hubble Space Telescope, 4 times HST resolution, and the last is at the point where the body roughly fills the field-of-view (FOV) and the phase angle is lowest. Data obtained in the Approach phase will provide a range of illumination angles to initialize the topographic model, and aid in finalizing the plans for HAMO and LAMO.

During the Vesta approach period several searches for hazards (dust, moons) will be performed in the far- and near-Vesta environment. During the Pre-Approach Cruise sub-phase, at a distance of approximately $1.4 \times 10^{6} \mathrm{~km}, 2 \times 2$ image mosaics will be obtained, covering the entire Hill sphere, optimized to detect potential satellites in orbits having periapsis above the Survey orbit. Additional observations will be made during the transit from Vesta Arrival (capture) to the Survey orbit with strategies optimized to detect potential satellites with orbits crossing the Survey, HAMO and LAMO orbits. VIR characterization of discovered satellites will help to define their relationship to specific regions of Vesta, as well as the mechanism and time of their ejection. Dust particles are most easily observed in forward-scattered light. Dawn approaches Vesta at a modestly high phase angle $\left(\sim 90^{\circ}\right)$, peaking at $\sim 105^{\circ}$ during the second rotation characterization map. It is likely that data obtained during the satellite searches can be used to search for dust in the vicinity of Vesta. Further analysis, including a search for stable orbits, is required before the details of hazards characterization observations can be planned at the activity level.

An additional activity in the Approach phase is to exercise the processing streams for the instruments data, mainly the FC and VIR, to verify that quicklook products can be produced on the required timelines, and to check and improve the calibration parameters.

\subsubsection{Vesta Survey Orbit}

The science goals for the Survey orbit are to obtain global coverage with VIR, and to create overlapping global images with the FC in all eight filters, centered every $20^{\circ}$ of longitude from the equator to the mid-latitudes $\left(\sim 50^{\circ}\right)$. In polar latitudes, the images will be taken at $40^{\circ}$ intervals. The VIR map constitutes the primary (and perhaps only) global spectral mapping. The FC maps will be 3-4 frames each. The dispersion in viewing angle across the camera field of view at the Survey altitude will provide global stereo coverage between adjacent overlapping sets of images. These VIR and FC global maps will be used for definition of targets to be investigated at lower altitudes, and the FC data will contribute significantly to the topographic model. Cross-calibration of the VIR and FC will be facilitated by concurrent imaging during this sub-phase.

\subsubsection{High Altitude Mapping Orbit}

The High Altitude Mapping Orbit (HAMO) is primarily used to create global FC maps of the lit surface in of the body in multiple filters from a nadir attitude, and two maps from two different off-nadir viewing angles, in the clear filter only. The Level-1 requirement is for $80 \%$ global coverage in 3 filters; we plan to acquire images of the entire lit surface in the clear plus seven filters. The three FC clear maps from different viewing angles (nadir plus two off-nadir) will be used to create a topographic model, using also limb images, via stereophotogrammetry and photoclinometry. The off-nadir coverage will be at angles of $10-20^{\circ}$ from the surface normal. 
VIR will also collect at least 5000 frames as part of the Level 1 requirements, but this will be far short of global coverage. The VIR frames will be collected concurrently with the FC data if possible. The VIR data will be collected to sample the spectral variability at smaller scales than the global Survey map, and to build up high-resolution coverage of areas of interest. The VIR data will be taken at nadir attitude; however, VIR data may also be acquired at off-nadir viewing angles, for which the scan mirror may be used to tailor the viewing angle when the geometry is favorable.

A second HAMO is planned near the end of the encounter, as the spacecraft is spiraling out from Vesta, to capture different lighting conditions, and to fill in gaps in the coverage. This activity is on the order of 3 days (TBC) and will complete global FC mosaics in multiple filters, including off-nadir views of the new illuminated polar region, and VIR spectra of selected targets and the lit pole.

The HAMO plan is the most constrained of any of the orbit sub-phases. It is constrained by the limitations of the data downlink volume, the need for systematic global coverage, and the need for resiliency to interruptions in the plan. The 26-day HAMO is split into two cycles, each 13 days in length, and each cycle is split into two campaigns. Each cycle achieves global coverage on a set of roughly evenly spaced ground tracks. The campaigns are delineated by the operational mode used for polar mapping (i.e., nadir versus fixed off-nadir versus general target pointing).

During the departure phase, the coverage begun in HAMO will be completed with nadir maps and off-nadir views of the newly illuminated polar region obtained concurrently by slewing, as the body-spacecraft relative motion is slow compared to the time needed to slew the spacecraft to obtain the off-nadir views. Gaps at low-latitude will be filled in an opportunistic manner.

\subsubsection{Low Altitude Mapping Orbit}

The purpose of LAMO is to obtain spatially resolved neutron and gamma ray spectra of the asteroid and global tracking coverage to determine the gravity field. The strategy is to be nadir pointed, with exceptions for limb imaging and limited target pointing. The bulk of the time will be spent pointed nadir, which is the attitude that GRaND needs to maximize their signal-to-noise. One $5.5 \mathrm{hr}$ downlink pass per day will obtain tracking data for gravity field determination. For both of these objectives, even ground track coverage is needed. The plan is for a repeating pattern of operations: nadir pointing observations, downlink pass, and orbit maintenance once every two days. There will be downlink to spare, so as much nadir imaging (FC and VIR) as can be fit into the data buffers will be obtained. Off-nadir targeted acquisition is anticipated for a small fraction of the time (likely $<10 \%$ ), including some very high resolution imaging of selected targets.

A dedicated gravity campaign (several days to one week) of continuous tracking is useful for resolving the wobble in the rotation to measure the moment of inertia of the protoplanets. One possibility is to have two 3-day continuous tracks that would empty buffers twice during LAMO (roughly after each third of the sub-phase), allowing more imaging science to be collected in the lowest altitude orbit.

\subsection{Mission Operations}

The Dawn mission operations will be managed by the Jet Propulsion Laboratory with the Dawn Science Center (DSC) at UCLA having the responsibility for the day-to-day coordination of science planning and sequencing activities. The Dawn mission is designed to follow heritage JPL Multi-Mission Ground Systems and Services (MGSS) philosophies and processes for mission operations, where feasible. MGSS has evolved over many years of operating JPL and contractor spacecraft, and has developed processes that are 


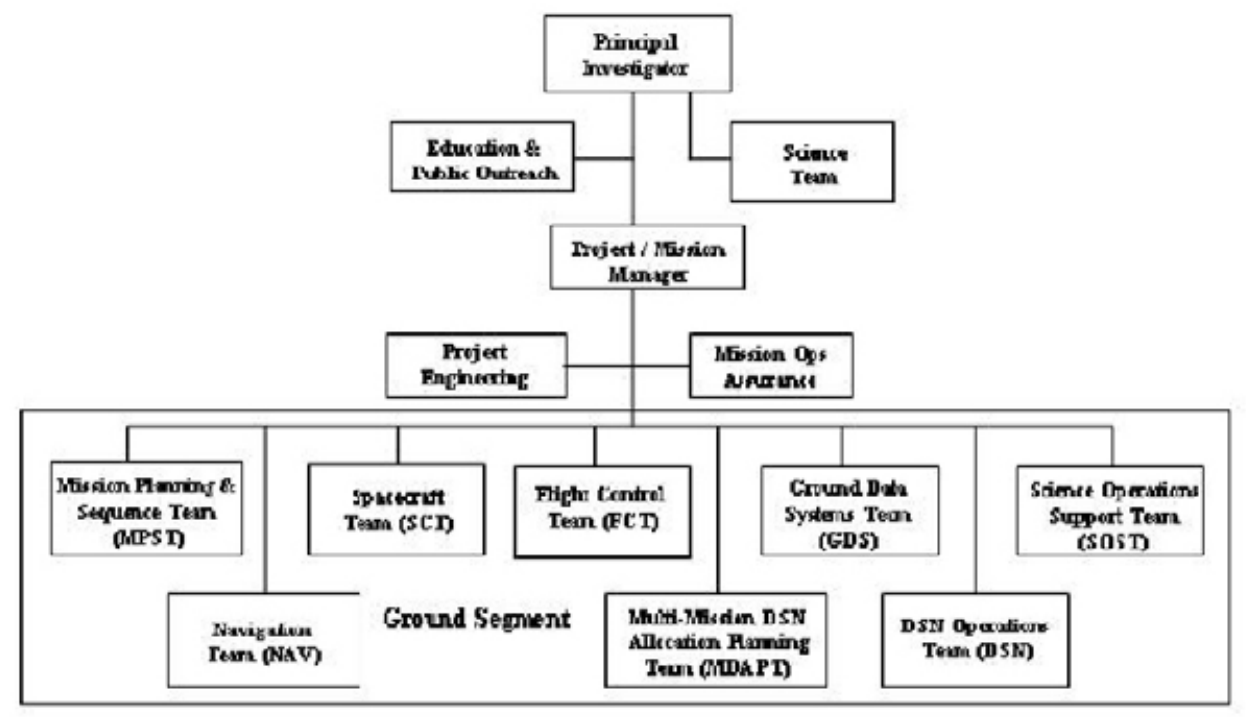

Figure 12. The Dawn Project operations organization showing the teams that are part of the Ground Segment. The Dawn Science Center is included within the SOST.

well formulated, well tested, and flight proven. These processes are adapted for each mission, and lessons learned are incorporated for each new mission. Fitting within the multi-mission operations framework was critical to keeping Dawn operations within the cost constraints of the Discovery program.

The Dawn project operations are the responsibility of the Ground Segment. The Ground Segment is divided into 3 operations organizations: the Navigation Team (NAV), the Mission Operations System (MOS), and the Science team. The NAV team is responsible for the formulation, estimation, and control of the flight systems interplanetary trajectory, as well as orbit determination and control after arrival at each of the asteroids. Prior to launch, the Mission Design and Navigation team is responsible for developing the project mission plan, consistent with the constraints imposed by the flight system design, the trajectory design, the navigation strategy, the science requirements, and the project requirements. The MOS is responsible for the collection of hardware, software, people, processes and procedures needed to operate the Dawn spacecraft from launch through the end of its mission. This same system is also used to support flight system testing during Assembly, Test and Launch Operations (ATLO). The Science team includes the Principal Investigator and all of the members of the project Science Team, as well as the Dawn Science Center, located at UCLA. The Science Team is responsible for planning science data collection, generating science commands and sequences, analyzing the returned science data, disseminating data and results to the broader science community, and the long-term archiving of returned science data. Figure 12 shows the Ground Segment as it is subdivided into the operations teams.

The Dawn project s Ground Segment operations are distributed and global. The Ground Segment infrastructure is encapsulated in the Ground Data System (GDS). The GDS architecture supports the distributed configuration and the interfaces required for flight operations. In effect, the GDS provides the ground-to-space link required to interface the ground system with the flight system. In addition, the GDS supports all mission development and test environments leading up to launch and into operations. 
These include the flight system testbeds at Orbital and JPL, ATLO at Orbital, Goddard Space Flight Center, and the Kennedy Space Center, Mission Support Areas at JPL and Orbital, Instrument Sites at DLR, MPS, INAF, ASI and LANL, and science operations at the Dawn Science Center at UCLA.

The Dawn Science Center (DSC) is the primary conduit for the flow of data and information between the Science and Instrument Teams and the MOS. The DSC is collocated with the principal investigator at UCLA so that the PI can provide guidance and assist in prioritizing science observations when conflicts arise due to resource limitations. It has conferencing facilities and office space to Science Team during high activity periods such as flybys and asteroid encounters. In addition to web-accessible computing and data distribution hardware. The DSC also houses a secure flight operations support area where science sequences are generated, validated and submitted to the project and raw downlink telemetry data are received, processed, and rebroadcast to the instrument teams over the public internet.

The operations teams that will be collocated at JPL include the Flight Control Team (FCT), the Mission Planning and Sequence Team (MPST), the Spacecraft Team (SCT), the Ground Data Systems Operations Team (GDST), the Multi-mission Deep Space Network Allocation and Planning Team (MDAPT), the Navigation Team (NAV), and the Science Operations Support Team (SOST). The GDS, NAV, MPST, and MDAPT teams include multi-mission support personnel who are not necessarily collocated with Dawn operations team members in the Mission Support Area. The SOST is the JPL interface the Dawn Science Center.

\subsubsection{Spacecraft Operations}

Although Orbital Sciences Corporation is building the flight system, spacecraft operations will be performed by a JPL Spacecraft Team following the post-launch initial checkout period. During Launch through initial spacecraft checkout, the SCT is an integrated distributed team between both JPL and Orbital. The Orbital spacecraft team will design the initial checkout activities and define the spacecraft flight rules and constraints to be followed once operations have been transitioned to JPL. This is one significant difference between Dawn and other JPL multi-mission projects where the spacecraft operations typically remain the responsibility of the spacecraft contractor.

\subsubsection{Navigation Planning and Analysis}

The Navigation (NAV) Team at JPL performs the orbit determination (OD) analysis and defines the IPS thrust profile to maintain the spacecraft trajectory to meet the mission objectives. During cruise, the OD analysis is performed with traditional radiometric navigation data (two-way Doppler and ranging) received from the Deep Space Network, once to twice weekly during cruise, and more frequently during initial checkout, the Mars Gravity Assist, and asteroid operations. During asteroid operations the radiometric data is supplemented with optical navigation data taken by the Framing Camera. During asteroid operations the NAV Team will make specific requests to the Framing Camera Team to acquire the images needed for optical navigation. The optical navigation data will be given a higher downlink priority over science imaging data to minimize delays in orbit determination.

As the flight system nears the asteroid, IPS thrusting is used to spiral in to the asteroid orbit. On final approach and during the early orbit phase, the navigation team will improve estimates of the asteroid mass, gravity field, and spin state in order to predict the trajectory and compute subsequent orbit correction maneuvers. The navigation team 
will update the orbit plan once the asteroid physical parameter estimates are adequately determined.

When in orbit about the asteroid, the navigation team relies on a combination of optical navigation and radiometric navigation. Typically, radio tracking will be available $24-$ 56 hours/week in science orbits, and 24 hours/week in transfers between those science orbits. Dedicated optical navigation data will be taken in approximately 1-hour sessions, once per day.

Navigation will develop a topographic map sufficient for navigation purposes, using a combined stereophotogrammetry/photoclinometry method that will approach a $10-\mathrm{m}$ accuracy for regions of the surface. The optical landmark tracking using in this method depends on knowledge of Framing Camera pointing and images of surface features (landmarks). Over time, specific landmarks observed in the images provide geometric information that is combined with the optical navigation data and/or radiometric data in the orbit determination filter.

\subsubsection{Mission Planning and Sequencing}

The Mission Planning \& Sequencing Team (MPST) is designed to support multiple projects in an efficient manner. By utilizing a combination of project dedicated and multi-mission personnel, the team is able to relatively easily shift resources to support the varying needs of a mission throughout their development and operations life cycles. In addition, the cross training that is required to support this approach, provides for a pool of well trained personnel, experienced in operations on multiple projects. The MPST will also be using a suite of multi-mission planning and sequencing tools that are being adapted for the Dawn Mission. The JPL MOS as well as the DSC at UCLA will use this tool set. The use of common tools simplifies the interface between the MOS and the DSC and minimizes costs.

The Dawn uplink process expands on the heritage uplink process used by other MGSS missions, with some adaptation for Dawn. The uplink process is guided by the Mission Plan and Science Plan both completed prior to launch, and updated 18 and 6 months, respectively prior to arrival at each asteroid. Instrument sequence and mission sequence plans are generated to define in detail sequence load boundaries and resource allocations, in order to be ready to build instrument sequences and science sequences for uplink. The fixed antenna on the Dawn spacecraft drives the need to select telecommunication passes with the Deep Space Network based on the geometry of the orbit and science objectives. Because the DSN is shared with other flight projects, requests for tracking coverage need to be requested far in advance, but whether or not they will be available will not be known with complete certainty until two months before they are used. Therefore, the science sequences need to be flexible enough to shift with changes in the DSN schedule. The science sequence generation is lead by the Dawn Science Center (DSC) and is described in the next section on Science Operations. The DSC will deliver the science sequences as conflict-free products ready to be processed into uplink products by the MPST, as well as other supporting products. Finally, the stored sequence is created by the MPST following a heritage MGSS process used for Odyssey and MGS, with minor changes for Dawn. The details of the spacecraft engineering activities will be added at this point along with the detailed specification of the spacecraft pointing.

All of the instruments are mounted directly to the spacecraft so the instrument pointing involves turning the spacecraft. The instruments also have radiators mounted on the $-\mathrm{X}$ side of the spacecraft and so all turns must be modeled to insure that they do not violate the sun constraints. Because the Dawn uplink process is success oriented and there is not 


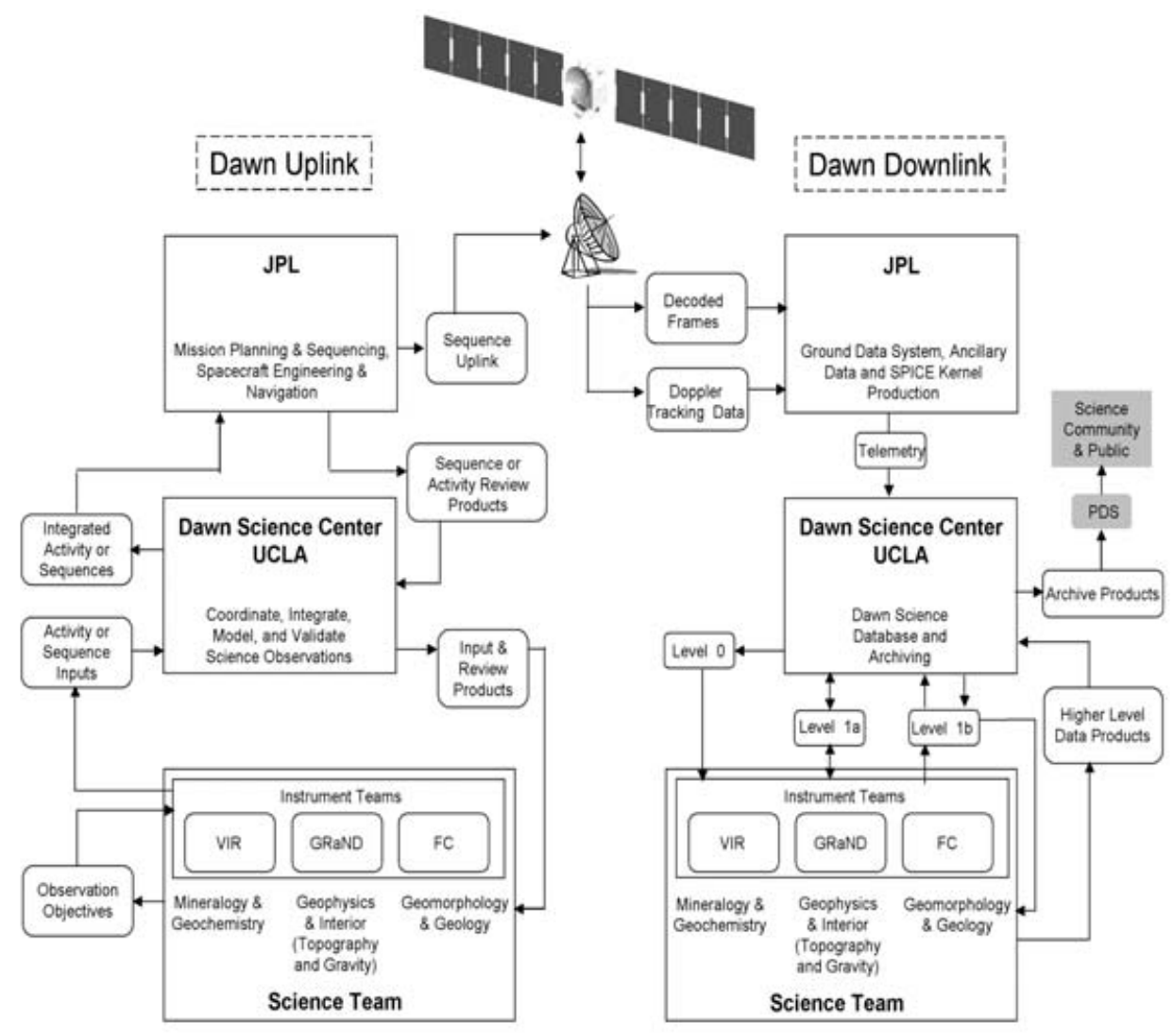

Figure 13. Dawn Uplink and Downlink process schematic. This diagram shows the interactions of the primary organizations associated with the Dawn uplink and downlink processes. Uplink products flow up the left-hand side of the diagram from the Science Team to the spacecraft and downlink products flow down the right-hand side.

time in the schedule for late changes, the science pointing strategies are being developed well in advance of the uplink process.

\subsection{Science Operations}

The DSC plays a central role in science planning and sequencing (uplink) and data distribution within the Science Team and the science community at large (downlink). Cost-capped missions such as Dawn cannot afford large mission operations teams. In order to work within the staffing limits, science observation strategies must be planned well in advance and only minimally revised during the science sequencing process to account for slight shifts in trajectory or timing. The left side of Figure 13 depicts the Dawn uplink process. The Science Team defines the science objectives and creates an observation plan to be implemented by the instrument teams. The instrument teams initially define observations (activities) to meet the objectives that are then passed to the DSC. The DSC integrates the activities and verifies that the integrated plan can be implemented within available spacecraft resources (memory buffers, downlink capability, etc.). Mission planners from the MPST review the plan to verify that sufficient margin remains 
for spacecraft engineering activities such as orbit maintenance, optical navigation, and downlink.

Several iterations are typically required in order to produce a plan that meets both the observation objectives and the spacecraft resource constraints. Once the observation plan is complete, the process is repeated at the instrument command level. The instrument teams design the commands sequences that are necessary to implement the observation plan for each mission phase. The DSC integrates and validates the sequences and produces review products. Once the science sequence has been accepted by the Science Team, it is integrated with the background spacecraft sequence and reviewed again prior to being sent to the spacecraft.

The right side of Figure 14 shows the downlink data flow process. Raw telemetry flows from the spacecraft, to the Deep Space Network (DSN), to the Dawn ground data system at JPL and then on to the DSC. Instrument housekeeping data that are returned in real-time are immediately rebroadcast to the instrument team home institutions for health and safety monitoring. Raw science data packets are stored in the Dawn Science Database (DSDb) after the completion of each DSN pass. Once the data become available, the instrument teams download the level 0 data (raw telemetry). After the data are decompressed, decoded, and formatted into scientifically useful data structures, the level-1a data products are uploaded back into the DSDb. Additional data processing is performed by the instrument teams to produce calibrated (level-1b) data products (radiometrically corrected images and spectra and fluxes from the GRaND instrument) which are then uploaded to the DSDb, along with data set descriptions, calibration data and instrument models, and calibration procedures. The Science Team uses the calibrated data acquired from the DSDb to produce derived data products (maps, mosaics, etc.) which are also deposited in the DSDb. The Dawn Science Center produces archive volumes (DVDs or other approved media) in compliance with PDS archiving standards and submits them to the PDS for archive and distribution to the science community and general public.

\subsubsection{Dawn Science Database}

The Dawn Science Database (DSDb) is a Dawn adaptation of technology developed by the Planetary Plasma Interactions (PPI) Node of the PDS. The MySQL database contains an inventory of external data files that includes metadata derived from the PDS labels associated with each file. The metadata are used to constrain queries that can locate individual files or collections of similar files. As a result, all products within the DSDb must be fully PDS compatible. All file management system systems require some form of metadata in order to perform their functions. The selection of the PDS metadata format for internal data tracking will reduce the cost and improve the efficiency of the downstream archive process.

The DSDb supports both file upload and download actions. All DSDb users are permitted to download any file within the inventory, however, only selected users are allowed to upload or update files. Once the user has constructed a query by selecting various options, a list of matching products is returned. Users may select none, any, or all of the products in the return set. The download process uses standard shopping cart technology to keep track of selected products. Once the user is finished, the contents of the shopping cart are zipped up and transferred to users workstation. Figure 15 shows the top level page of the DSDb after the user has been authenticated and user privileges determined. The web interface to the DSDb is dynamically generated based on user privileges so that users are only presented with options that they are authorized to perform. The view of the Uplink pages shown in Figure 14 only appears to users with instrument manager 


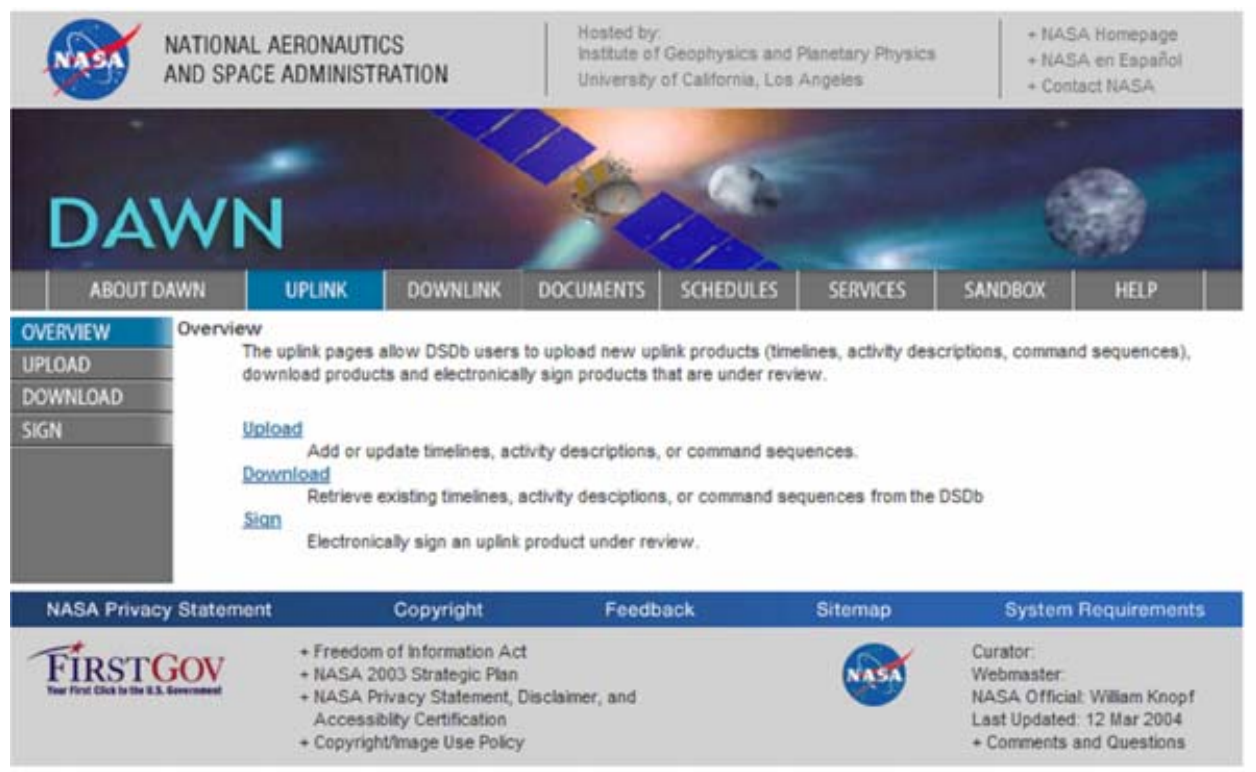

Figure 14. Dawn Uplink and Downlink process schematic. This diagram shows the interactions of the primary organization

privileges. The PDS labels for data products, calibration files, etc. that are required by the DSDb for inventory tracking are uploaded with the products. Many of these products use PDS labels that are physically attached to the data files. PDS labels for other products such as sequences, documents, and presentations are generated automatically after the user enters a few required parameters through the web interface. Raw telemetry data files (level 0) are labeled by a separate automated process at the time they are created.

\subsubsection{Archive Validation, Generation and Schedule}

The primary mechanism by which data products will be validated is through usage by the Dawn Science Team. All products used by the Science Team for analysis or to produce higher level data products are acquired from the DSDb. Errors that are uncovered by users are reported back to the data provider through the DSDb. When corrected files are uploaded to the DSDb the original files are removed from the inventory.

Table 3 gives the basic Dawn data internal distribution and archive schedule. In general, raw telemetry data are provided to the team within 6 hours of each downlink pass and submitted to the PDS to be saved at the end of each mission phase. Raw (level 1a) and calibrated (level 1b) data are then returned by the teams to the DSDb weekly. Raw data are submitted to the PDS for archive at 3 months after the end of each mission phase. Calibrated data are archived within 6 months after the end of each asteroid encounter. This additional delay is needed in order to validate the instrument calibrations. Finally, higher level data products (maps, mosaics, models) must be uploaded into the DSDb within 10 months after departure. This allows the Science Team approximately 2 months to review these products before they are submitted to the PDS for archive. For Ceres, we plan to reduce these delivery times by a factor of two (as indicated in Table 3 ) in order to archive the data before the end of mission is reached, which occurs 5 months after Ceres departure. This timeline is reasonable because the team will have gained experience with the Vesta deliveries. 
Table 3. Dawn data products, processing levels and availability

\begin{tabular}{|c|c|c|c|}
\hline $\begin{array}{l}\text { Processing } \\
\text { Level }\end{array}$ & Product description & $\begin{array}{l}\text { Available to } \\
\text { Science Team }\end{array}$ & PDS Archive \\
\hline Level 0 & Raw telemetry data & 6 hrs after DSN pass & $\begin{array}{l}\text { End of mission } \\
\text { phase }\end{array}$ \\
\hline Level 1a & $\begin{array}{l}\text { Raw data (data numbers) reformatted } \\
\text { into images, spectral cubes, } \\
\text { time-series }\end{array}$ & $\begin{array}{l}\text { Within } 7 \text { days of } \\
\text { downlink }\end{array}$ & $\begin{array}{l}3 \text { months after } \\
\text { end of phase }\end{array}$ \\
\hline Level 1b & $\begin{array}{l}\text { Radiometrically calibrated images } \\
\text { and spectra, gamma ray and neutron } \\
\text { count rate time-series }\end{array}$ & $\begin{array}{l}\text { Within } 14 \text { days of } \\
\text { downlink }\end{array}$ & $\begin{array}{l}6(3)^{1} \text { months after } \\
\text { asteroid departure }\end{array}$ \\
\hline Level 2 & $\begin{array}{l}\text { Geometrically corrected and co- } \\
\text { registered image and spectral, } \\
\text { gridded gamma ray and neutron } \\
\text { count rates, gravity coefficients } \\
\text { and uncertainties }\end{array}$ & $\begin{array}{l}\text { Within } 6(3) \text { months } \\
\text { of asteroid departure }\end{array}$ & $\begin{array}{l}12(6) \text { months after } \\
\text { asteroid departure }\end{array}$ \\
\hline Level 3 & $\begin{array}{l}\text { Global clear and colored atlases and } \\
\text { global mosaic, Olivine and Pyroxene } \\
\text { abundance maps, Elemental abundance } \\
\text { maps, Free-air gravity map, geoid } \\
\text { and uncertainty map }\end{array}$ & $\begin{array}{l}\text { Within } 8(4) \text { months } \\
\text { of asteroid departure }\end{array}$ & $\begin{array}{l}12(6) \text { months after } \\
\text { asteroid departure }\end{array}$ \\
\hline Level 4 & $\begin{array}{l}\text { Topographic model, Geologic map, } \\
\text { Bouguer gravity map }\end{array}$ & $\begin{array}{l}\text { Within } 10(5) \\
\text { months of asteroid } \\
\text { departure }\end{array}$ & $\begin{array}{l}12(6) \text { months after } \\
\text { asteroid departure }\end{array}$ \\
\hline
\end{tabular}

1) Numbers in parentheses pertain to Ceres

Dawn data will undergo an additional set of reviews associated with the PDS archive process. Prior to launch, the design of the standard data products (levels 1a, 1b, and 2) will each be formalized in an interface control document (ICD) that will be subject to PDS review and signature. Once the archive design documents are signed, archive volumes will be generated using data from the instrument checkout and the initial in-flight calibration tests. These data will undergo a full PDS peer review and lien resolution process. The peer review panel will verify the data are scientifically useful and adequately documented, and that the data formats are usable to the science community at large. Once this process is complete, the Dawn archive design will be under configuration management and any design changes will require an additional external review. The final validation procedure is to verify that the data sets submitted to PDS conform to the standards set forth in the archive design specification.

\section{Concluding Remarks}

Dawn makes the first attempt to explore the nebular compositional gradient by exploring two bodies on either side of the dew/frost line in the early solar system. Ion propulsion is the technology that enables this advance in our exploration of the solar system. The spacecraft is nearing completion and plans are well underway to operate the spacecraft and return and archive the data needed to achieve the scientific objectives. Launching a mission of this size is a complex, but very worthwhile undertaking. Discovery missions provide good value for the expenditures required. Nevertheless Dawn is only the first step. There is much left to do in exploring the minor planets. 


\section{Acknowledgements}

The success of the Dawn project has been

due to the hard work of many individuals at Orbital Sciences Corporation, the Jet Propulsion Laboratory and their subcontractors. In addition the Dawn Science Team has played an essential role in designing the mission and supplying the payload. We are extremely grateful for their help. This work was supported by the Jet Propulsion Laboratory under contract to the National Aeronautics and Space Administration.

\section{References}

Binzel, R.P., Gaffey, M.J., Thomas, P.C., Zellner, B.H., Storrs, A.D., Well, E.N. 1997, Icarus 128,95

Drummond, J.D., Fugate, R.Q., Christou, J.C. 1998, Icarus 132, 80

Kuzmanoski, M. \& Kovacevic, A. 2002, Astron. \& Astro. 395, L17

Li, J-Y., McFadden, L.A., Parker, J.W., Young, E.F., Stern, S.A., Thomas, P.C., Russell, C.T., Sykes, M.V. 2005, Icarus submitted

McCord, T.B. \& Sotin, C. 2005, J. Geophys. Res. 110, DOI:10.1029/2004JE002377, E05009-1E05009-14

Russell, C.T., Capaccioni, F., Coradini, A., De Sanctis, M.C., Feldman, W.C., Jaumann, R., Keller, H.U., McCord, T.B., McFadden, L.A., Mottola, S., Pieters, C.M., Prettyman, T.H., Raymond, C.A., Sykes, M.V. Smith, D.E., Zuber, M.T. 2005, in: Solar System Small Bodies: Synergy between In Situ and Remote Observations, (Springer)

Thomas, P.C., Parker, J.W., McFadden, L.A., Russell, C.T., Stern, S.A., Sykes, M.V., Young, E.F. 2005 Nature 437, 224

Treiman, A.H., Lanzirotti, A. \& Xirouchakis, D. 2004 Earth Planet Sci. Lett. 219, 189 